\title{
REPRESENTATION OF FUNCTIONS BY MATRICES. APPLICATION TO FABER POLYNOMIALS
}

\author{
ERI JABOTINSKY
}

Introduction. Let $\Omega$ be the family of functions $f(z)$ such that for $|z|<\rho$ with $\rho>0$

$$
f(z)=\sum_{n=1}^{n=+\infty} f_{n} z^{n} \quad\left(\text { with } f_{1} \neq 0\right) .
$$

Raising (1) to the $m$ th power ( $m$ integer, $-\infty<m<+\infty$ ) we can always find coefficients $f_{m, n}$ such that for $|z|<\rho^{\prime}, \rho^{\prime}>0$,

$$
[f(z)]^{m}=\sum_{n=-\infty}^{n=+\infty} f_{m, n} z^{n}
$$

We note that because $f(z) \in \Omega$, we have $f_{m, n}=0$ for $n<m$.

The matrix $f=\left\|f_{m, n}\right\|$ is a transform of the function $f(z)$ and can be used to represent this function. One row of the matrix is sufficient to define the function so that the whole matrix furnishes a superabundance of information about the function. This results, however, in the existence of relations among the elements $f_{m, n}$ which are independent of the particular function $f(z)$. These relations are fundamental properties of all matrices $f$ which represent functions $\in \Omega$ and they are powerful analytical tools that can be applied to many problems of analysis.

We shall prove two such fundamental theorems about the matrices $f=\left\|f_{m, n}\right\|$ and then, as an application, use these theorems to derive properties of Faber polynomials which play an important role in the theory of univalent functions.

Historical. Integral transforms in two variables, generalizing the matrix transforms which are considered here, were defined, and some of their properties were given by the author in the summary of a paper submitted by the author to the 1950 Congress of Mathematicians at Harvard.

I. Schur $[5]^{1}$ considered in connection with functions $f(z)$ the matrices $f_{+}=\left\|f_{m, n}\right\|$ defined for $m, n \geqq 1$. His failure to consider the full matrix $f=\left\|f_{m, n}\right\|$ defined for $-\infty<m, n<+\infty$ prevented his dis1952.

Received by the editors December 4, 1951 and, in revised form, September 2,

${ }^{1}$ Numbers in brackets refer to the bibliography at the end of the paper. 
covery of our Theorem II. The matrices $f_{+}$were also used by $\mathbf{M}$. Schiffer [4] and by the author [2].

Theorem II was first proved by the author [3] by contour integration. An algebraic proof based on the fact that $f_{m, n}$ is a polynomial in $m$ of degree $(n-m)$ was indicated. The possibility of the present proof was suggested by $M$. Schiffer who also found a proof based on the study of the expression $\log [(f(z)-f(w)) /(z-w)]$ (see our proof of Theorem VIII). Thus, in all, four proofs of Theorem II are available. The first proof by contour integration has the advantage of being adaptable to functions not in $\Omega$ and to general integral transforms of the type (1).

Grunsky's theorem (our Theorem VII) was first proven by Grunsky by contour integration [1]. Grunsky's theorem results from our Theorem II but is weaker than this theorem.

The polynomials $F_{m}^{*}(t)=(1 / m) F_{m}^{\prime}(t)$ were used by $M$. Schiffer in preference to the Faber polynomials $F_{m}(t)$ in connection with the coefficient problem of uniform functions [4]. Equation (13) of our Theorem $\mathrm{V}$ shows that these polynomials are in a sense covariant with the $f_{m, n}$ when $f(z)$ is replaced by $f\{g(z)\}$. This explains the preference.

Two fundamental theorems. Using the notation introduced in (2), we have the following general properties of the matrices $f=\left\|f_{m, n}\right\|$ :

Theorem I. Consider two functions $f(z) \in \Omega$ and $g(z) \in \Omega$ and the function $k(z)=f\{g(z)\}$ which is also $\in \Omega$. Let $f, g$ and $k$ be matrices representing these functions. Then $k=f \times g$. That is,

$$
k_{m, n}=\sum_{p=-\infty}^{p=+\infty} f_{m, p} g_{p, n} .
$$

ThEOREM II. If $f(z) \in \Omega$ and $\phi(z)$ is the inverse function of $f(z)$, then $\phi(z) \in \Omega$. Let $f$ and $\phi$ be the matrices representing $f(z)$ and $\phi(z)$. Then the elements $\phi_{m, n}$ of $\phi$ are given by

$$
\phi_{m, n}=\frac{m}{n} f_{-n,-m} \quad(\text { for } n \neq 0)
$$

and, for $n=0$, by

$$
\sum_{m=-\infty}^{m=-\infty} \phi_{m, 0} z^{-m-1}=f^{\prime}(z) / f(z) .
$$

Proof of Theorem I. We identify the coefficients of $z^{n}$ in the second and last terms of the following equation: 


$$
\begin{aligned}
{[k(z)]^{m} } & =\sum_{n=-\infty}^{n=+\infty} k_{m, n} z^{n}=[f\{g(z)\}]^{m}=\sum_{p=-\infty}^{p=+\infty} f_{m, p}[g(z)]^{p} \\
& =\sum_{p=-\infty}^{p=+\infty} f_{m, p}\left[\sum_{n=-\infty}^{n=+\infty} g_{p, n} z^{n}\right] \\
& =\sum_{n=-\infty}^{n=+\infty}\left[\sum_{p=-\infty}^{p=+\infty} f_{m, p} g_{p, n}\right] z^{n} .
\end{aligned}
$$

The change in the order of summation is in our case always legitimate because the sum $\sum_{p=-\infty}^{p=+\infty} f_{m, p} g_{p, n}$ contains only a finite number of significant terms (those for which $m \leqq p \leqq n$ ).

Proof of Theorem II. We use the fact that $f\{\phi(z)\}=z$ and Theorem I. Noting that the matrix $I$ representing the function $z$ is the unit matrix $I=\left\|\delta_{m, n}\right\|$, we have

$$
\sum_{p=-\infty}^{p=+\infty} f_{m, p} \phi_{p, n}=\delta_{m, n}
$$

The matrix $f$ is not singular and has only one inverse $\phi$. All we have to do is to show that the values of $\phi_{m, n}$ drawn from (4) and (5) satisfy identically (6). We show first that (4) and (5) are together equivalent to

$$
\sum_{m=-\infty}^{m-\infty} \phi_{m, n} z^{-m-1}=[f(z)]^{-n-1} \cdot f^{\prime}(z)
$$

Indeed, for $n=0$ this is clear. For $n \neq 0$ we have

$$
[f(z)]^{-n-1} \cdot f^{\prime}(z)=-\frac{1}{n} \frac{d}{d z}[f(z)]^{n}=-\frac{1}{n} \sum_{p=-\infty}^{p=+\infty} p f_{-n, p} z^{p-1}
$$

which for $p=-m$ shows that, for $n \neq 0,(7)$ and (4) are equivalent.

Consider now the product

$$
[f(z)]^{m} \cdot[f(z)]^{-n-1} \cdot f^{\prime}(z)=\left[\sum_{p=-\infty}^{p=+\infty} f_{m, p} z^{p}\right]\left[\sum_{p=-\infty}^{p=+\infty} \phi_{p, n} z^{-p-1}\right] .
$$

The coefficient of $z^{-1}$ on the right is $\sum_{p=-\infty}^{p=+\infty} f_{m, p} \phi_{p, n}$; on the left we have:

$$
[f(z)]^{m} \cdot[f(z)]^{-n-1} \cdot f^{\prime}(z)=[f(z)]^{m-n-1} \cdot f^{\prime}(z) .
$$

If $m=n$, the coefficient of $z^{-1}$ is 1 . If $m \neq n$, then the last expression is equal to $(1 /(m-n)) d[f(z)]^{m-n} / d z$ and the coefficient of $z^{-1}$ is 0 . Thus (6) is always satisfied. 
Faber polynomials. Let $f(z) \in \Omega$. We define for $m \geqq 1$ the $m$ th Faber polynomial of $f(z)$ as the polynomial $F_{m}(t)=\sum_{p=0}^{p=m} F_{m, p} t^{p}$ of degree $m$ for which

$$
F_{m}\left[\{f(z)\}^{-1}\right]=z^{-m}+\sum_{n=1}^{n=+\infty} c_{m, n} z^{n}
$$

That this defines the Faber polynomials uniquely can be shown by recurrence over $m$. We prove the following theorems:

THEOREM III. The mth Faber polynomial of the function $f(z)$ is

$$
F_{m}(t)=\phi_{-m, 0}+\sum_{p=1}^{p=m} \frac{m}{p} f_{p, m} t^{p} .
$$

TheOREM IV. Let $F_{m}(t)$ be the mth Faber polynomial of $f(z)$ and let $F_{m}^{*}(t)$ be the polynomial defined by

$$
F_{m}^{*}(t)=\frac{1}{m} F_{m}^{\prime}(t)
$$

Then

$$
F^{*}(t)=\sum_{p=1}^{p=m} f_{p, m} t^{p-1}
$$

Theorem V (Change of base). Let $f(z) \in \Omega$ and $g(z) \in \Omega$. Then $k(z)=f\{g(z)\}$ is also $\in \Omega$. Let $F_{m}(t)=\sum_{p=0}^{p=m} F_{m, p} t^{p}, G_{m}(t)$ $=\sum_{p=0}^{p=m} G_{m, p} t^{p}$, and $K_{m}(t)=\sum_{p=0}^{p=m} k_{m, p} t^{p}$ be the mth Faber polynomials of $f(z), g(z)$, and $k(z)$. And let $F_{m}^{*}(t)=(1 / m) F_{m}^{\prime}(t), G_{m}^{*}(t)=(1 / m) G_{m}^{\prime}(t)$, and $K_{m}^{*}(t)=(1 / m) k_{m}^{\prime}(t)$. Then the following relations hold:

$$
\begin{aligned}
K_{m, n} & =\sum_{p=n}^{p=m} G_{m, p} F_{p, n}, \\
K_{m}^{*}(t) & =\sum_{q=1}^{q=m} g_{q, m} F_{q}^{*}(t) .
\end{aligned}
$$

THEOREM VI (Generating functions). We have, respectively,

$$
\frac{x f^{\prime}(x)}{f(x)} \cdot \frac{1}{1-t f(x)}=1+\sum_{m=1}^{m-\infty} F_{m}(t) x^{m}
$$

and

$$
\frac{f(x)}{1-t f(x)}=\sum_{m=1}^{m-+\infty} F_{m}^{*}(t) x^{m} .
$$


THEOREM VII (Grunsky's theorem). We have in (8):

$$
n c_{m, n}=m c_{n, m} .
$$

TheOREM VIII (Schiffer's theorem). We have, for $f(z) \in \Omega$ :

$$
\log \frac{f(w)-f(z)}{w-z}=-\log f_{1,1}+\log \frac{f(w)}{w}
$$

$$
+\log \frac{f(z)}{z}-\sum_{m, n=1}^{m, n=+\infty} \frac{1}{m} c_{m, n} w^{m} z^{n}
$$

Proof of Theorem III. We have, for $m \geqq 1$,

$$
\begin{aligned}
\sum_{p \rightarrow 0}^{p-m} \phi_{-m,-p}[f(z)]^{-p} & =\sum_{p=0}^{p=-m} \phi_{-m,-p}\left[\sum_{n=-\infty}^{n=+\infty} f_{-p, n} z^{n}\right] \\
& =\sum_{n=-\infty}^{n-\infty}\left[\sum_{p=0}^{p=m} \phi_{-m,-p} f_{-p, n}\right] z^{n} .
\end{aligned}
$$

But

$$
\sum_{p \rightarrow-\infty}^{p=+\infty} \phi_{-m,-,} f_{-p, n}=\sum_{p=-n}^{p=m} \phi_{-m,-p} f_{-p, n}=\delta_{-m, n .}
$$

Whence, for $n \leqq 0$,

$$
\sum_{,=0}^{p=m} \phi_{-m,-p} f_{-p, n}=\delta_{-m, n}
$$

Therefore

$$
\sum_{p=0}^{p-m} \phi_{-m,-p}[f(z)]^{-p}=z^{-m}+\sum_{n=1}^{n-+\infty}\left[\sum_{p=0}^{p-m} \phi_{-m,-p} f_{-p, n}\right] z^{n},
$$

which proves that the polynomial $F_{m}(t)=\sum_{\substack{p=0 \\ p=0}} \phi_{-m,-p} t^{p}$ is the $m$ th Faber polynomial of $f(z)$, whence, using (4), we get (9). Incidentally, comparing equation (18) to equation (8) and noting that, for $n \geqq 1$, $f_{0, n}=0$, we also find that, for $m, n \geqq 1$ :

$$
c_{m, n}=\sum_{p=1}^{p-m} \phi_{-m,-p} f_{-p, n} .
$$

Proof of Theorem IV. We have (11) from (10) by differentiating (9) and dividing by $m$.

Proof of Theorem V. Let $\phi(z), \gamma(z)$, and $k(z)$ be the inverse functions of $f(z), g(z)$, and $k(z)$. Then, by Theorem III, equation 
(12) is equivalent to

$$
\kappa_{-m,-n}=\sum_{p=n}^{p=m} \gamma_{-m,-p} \phi_{-p,-n} .
$$

But this results from $\kappa(z)=\gamma\{\phi(z)\}$ and Theorem I. To prove (13) we have to show that $\sum_{p=1}^{p-m} k_{p, m} t^{p-1}=\sum_{q=1}^{a-m} g_{q, m}\left[\sum_{p=1}^{p=q} f_{p, q} t^{p-1}\right]$ or that $k_{p, m}=\sum_{q=p}^{q=m} f_{p, q} g_{q, m}$, which results from Theorem I.

Proof of Theorem VI. We first prove (14). We have:

$$
\begin{aligned}
\frac{f(x)}{1-t f(x)} & =\sum_{p=1}^{p=+\infty}[f(x)]^{p} t^{p-1}=\sum_{p=1}^{p-+\infty}\left[\sum_{m=p}^{m-+\infty} f_{p, m} x^{m}\right] t^{p} \\
& =\sum_{m=1}^{m=+\infty}\left[\sum_{p=1}^{p=m} f_{p, m} t^{p-1}\right] x^{m}
\end{aligned}
$$

which, by (11), proves (15).

To prove (14) we write (15) as

$$
f(x) /[1-t f(x)]=\sum_{m=1}^{m=+\infty} \frac{1}{m} F_{m}^{\prime}(t) x^{m}
$$

and integrate it with respect to $t$. We find

$$
-\log [1-t f(x)]=\sum_{m=1}^{m=+\infty} \frac{1}{m} F_{m}(t) x^{m}+g(x)
$$

where $g(x)$ is introduced by the integration. We now derive with respect to $x$ and find:

$$
\frac{t f^{\prime}(x)}{1-t f(x)}=\sum_{m=1}^{m-\infty} F_{m}(t) x^{m-1}+g^{\prime}(x) .
$$

Putting $t=0$ and noting that $F_{m}(0)=\phi_{-m, 0}$ we find

$$
0=\sum_{m=1}^{m-\infty} \phi_{-m, 0} x^{m-1}+g^{\prime}(x) \text {. }
$$

But, by (5), $\sum_{m=0}^{m-+\infty} \phi_{-m, 0} x^{m-1}=f^{\prime}(x) / f(x)$. Hence

$$
g^{\prime}(x)=-f^{\prime}(x) / f(x)+1 / x .
$$

Putting this into (20) and multiplying by $x$ we prove (14). We note that the expression for (14) may make it desirable to put, by definition, $F_{0}(t)=1$, a definition in conformity with (8) and (9). One would then put $F_{0}^{*}(t)=0$, a definition not in contradiction with (10) and (11). 
Proof of Theorem VII. We have, remembering that, in (8), $m, n \geqq 1$,

$$
\begin{aligned}
\sum_{p=-\infty}^{p=+\infty} \phi_{-m,-p} f_{-p, n}= & \sum_{p=-\infty}^{p=-1} \phi_{-m,-p} f_{-p, n}+\phi_{-m, 0} f_{0, n} \\
& +\sum_{p=1}^{p=+\infty} \phi_{-m,-p} f_{-p, n}=\delta_{-m, n} .
\end{aligned}
$$

But $f_{0, n}=0$ for $n>0$ and $\delta_{-m, n}=0$ because $-m<0$ and $n>0$. Therefore, changing $p$ into $-q$ in the first sum, we have

$$
\sum_{q=1}^{q=+\infty} \phi_{-m, q} f_{q, n}+\sum_{p=1}^{p=+\infty} \phi_{-m,-p} f_{-p, n}=0
$$

Using (4), put $\phi_{-m, q}=-(m / q) f_{-q, m}$ and $f_{q, n}=(q / n) \phi_{-n,-q}$ in the first sum. The above equation becomes:

$$
\sum_{q=1}^{q=+\infty}-\frac{m}{n} \phi_{-n,-q} f_{-q, m}+\sum_{p=1}^{p=+\infty} \phi_{-m,-p} f_{-p, n}=0
$$

or, according to (19),

$$
-(m / n) c_{n, m}+c_{m, n}=0,
$$

which proves the theorem.

Proof of TheOREM VIII. Multiplying both sides of (8) by $w^{m}$ and summing over $m$, we find, using (14),

$$
\frac{w f^{\prime}(w)}{f(w)} \cdot \frac{1}{1-f(w) / f(z)}-1=\sum_{m=1}^{m-\infty} w^{m} z^{-m}-\sum_{m, n=1}^{m, n=+\infty} c_{m, n} w^{m} z^{n},
$$

or

$$
\frac{f^{\prime}(w)}{f(w)} \cdot \frac{f(z)}{f(z)-f(w)}-\frac{1}{w} \cdot \frac{1}{z-w}=-\sum_{m, n=1}^{m, n=+\infty} c_{m, n} w^{m} z^{n},
$$

which can be written

$$
-\frac{f^{\prime}(w)}{f(w)}+\frac{f^{\prime}(w)}{f(z)-f(w)}+\frac{1}{w}-\frac{1}{z-w}=-\sum_{m, n=1}^{m, n=+\infty} c_{m, n} w^{m} z^{n} .
$$

Integrating over $w$ we find:

$$
\log \frac{f(w)-f(z)}{w-z}-\log \frac{f(w)}{w}-g(z)=-\sum_{m, n=1}^{m, n=+\infty} c_{m, n} w^{m-1} z^{n}
$$

where $g(z)$ is introduced by integration. Putting $w=0$ we find: 


$$
\log \frac{f(z)}{z}-\log f_{1,1}-g(z)=0
$$

which proves the theorem.

Noting that we have $\log \left((f(w)-f(z) /(w-z))=\sum_{m, n=0}^{m, n-\infty} d_{m, n} w^{m} z^{n}\right.$ with $d_{m, n}=d_{n, m}$ in the vicinity of $w=z=0$, and expanding the lefthand side explicitly in a double power series in $w$ and $z$, it can be shown that if $\phi_{m, n}$ be defined by (4) and (5), then $\sum_{p=-\infty}^{p=+\infty} \phi_{m, n} f_{p, n}$ $=\delta_{m, n}$, which furnishes an alternative proof of Theorem II, due to M. Schiffer.

\section{BIBLIOGRAPHY}

1. H. Grunsky, Koeffizientenbedingungen für Schlichtabbildende meromorphe Funktionen, Math. Zeit. vol. 45 (1939) pp. 29-61.

2. E. Jabotinsky, Sur la representation de la composition de fonctions par un produit de matrices. Application d l'itération de es et de $e^{z}-1$, C. R. Acad. Sci. Paris vol. 224 (1947) pp. 323-324.

3. - Sur les fonctions inverses, C. R. Acad. Sci. Paris vol. 229 (1949) pp. 508-509.

4. M. Schiffer, Faber polynomials in the theory of univalent functions, Bull. Amer. Math. Soc. vol. 54 (1948) pp. 503-517.

5. I. Schur, On Faber polynomials, Amer. J. Math. vol. 67 (1945) pp. 33-41.

JERUSALEM, ISRAFI 